\title{
Temperature dependent optical conductivity of undoped cuprates with weak exchange
}

\author{
J. Málek, ${ }^{1,2}$ S.-L. Drechsler*, ${ }^{1}$ U. Nitzsche, ${ }^{1}$ H. Rosner, ${ }^{3}$ and H. Eschrig ${ }^{1}$ \\ ${ }^{1}$ Leibniz-Institut für Festkörper- und Werkstoffforschung Dresden, P.O. Box 270116, D-01171 Dresden, Germany \\ ${ }^{2}$ Institute of Physics, ASCR, Na Slovance 2, CZ-18221 Praha 8, Czech Republic \\ ${ }^{3}$ Max-Planck-Institut für Chemische Physik fester Stoffe, Dresden, Germany
}

(Dated: November 5, 2018)

\begin{abstract}
The optical conductivity $\sigma(\omega)$ is calculated at finite temperature $T$ for $\mathrm{CuO}_{2}$ chain clusters within a $p d$-Hubbard model. Data at $T=300 \mathrm{~K}$ for $\mathrm{Li}_{2} \mathrm{CuO}_{2}$ are reanalyzed within this approach. The relative weights of Zhang-Rice singlet and triplet charge excitations near 2.5 and $4 \mathrm{eV}$, respectively, depend strongly on $T$, and a rather dramatic dependence of $\sigma(\omega)$ on the ratio of the $1^{\text {st }}$ to $2^{\text {nd }}$ neighbor exchange integrals is predicted. On the basis of these results, information about exchange interactions for frustrated edge-shared cuprates can be obtained from $T$-dependent optical spectra. Our results are also relevant for magnetically weakly coupled wide-gap insulators in general.

PACS numbers: 71.15.Mb, 71.27.+a, 74.72.Jt, 75.10.Jm, 75.10.Pq, 78.20.-e
\end{abstract}

Standard wisdom on wide gap insulators says that their optical spectra above $1 \mathrm{eV}$ excitation energy are hardly affected by temperature $T$, magnetic fields $H$, and by the magnetic nature of their ground state (GS). Moreover, spin and charge degrees of freedom are often decoupled in one dimension (1D). Here, we present exact theoretical results qualitatively valid for several cuprates to be specified below which prove just the opposite in all these respects. Our study bases on the fact that different magnetic states with different symmetries obey different selection rules, which - in case of soft magnetic materials - can lead to sizable $T$-dependence of the optical spectra. For systems with small enough exchange integrals, the energy difference between the GS and the excited magnetic states of the system will be small enough so that they can be thermally populated. This will cause a strong $T$-dependence of various response functions, here presented for the case of the optical conductivity $\sigma(\omega)$. For an illustration of our approach we consider $\mathrm{Li}_{2} \mathrm{CuO}_{2}$ being of current interest $1,2,3,4,5,6,7,8,9,10,11,12$, structurally simple, and most importantly, where single crystal data are available in a broad $\omega$-range 1,2 . This system stands for a class of frustrated spin- $1 / 2$ chain materials with both small ferromagnetic (FM) NN Cu-Cu exchange coupling $J_{1}$ and AFM next-nearest neighbor $(\mathrm{NNN}) \mathrm{Cu}-\mathrm{Cu}$ in-chain exchange $J_{2} \frac{1,3}{3}$ in terms of a 1D spin- $1 / 2$ Heisenberg model

$$
\mathcal{H}_{\mathrm{S}}=\sum_{i} J_{1} \mathbf{S}_{i} \mathbf{S}_{i+1}+J_{2} \mathbf{S}_{i} \mathbf{S}_{i+2}+J_{3} \mathbf{S}_{i} \mathbf{S}_{i+3}+\ldots
$$

In $\mathrm{Li}_{2} \mathrm{CuO}_{2}$ chains running along the $b$-axis are formed by the edge-sharing of $\mathrm{CuO}_{4}$ plaquettes. The most exciting puzzle addressed here is the missing of the ZhangRice singlet peak (ZRS) in reflectivity and electron energy loss spectroscopy (EELS) data at $T=300 \mathrm{~K}^{1,2}$. Its detection in resonant inelastic x-ray scattering (RIXS) spectra is under debate, too $\underline{4.5}$. There is also no consensus on the role of two scenarios for the FM in-chain order below the Néel temperature $T_{N}=9 \mathrm{~K}$. Scenario I is given by a dominant FM $J_{1}{ }^{\underline{9}}$ defined by the inequality $\alpha=-J_{2} / J_{1}<\alpha_{c} \approx 0.25$, if $J_{3}$ is weak ${ }^{13}$, where $\alpha_{c}$ marks the transition from a high-spin FM to a lowspin spiral-like ground state (GS). While in scenario II it stems from a specific AFM inter-chain coupling and a single chain would be at $\alpha>\alpha_{c} \underline{1,4,14}$.

A first microscopic theoretical description $\frac{1}{2}$ of the magnetic susceptibility $\chi(T)$ and of $\sigma(\omega)$ at zero temperature on the basis of a $\mathrm{Cu} 3 d_{x y} \mathrm{O} 2 p_{x, y}$ Hubbard model $(x$ and $y$ along the $b$ - and $c$-axes, respectively, see Figs. 1,2 of Ref. 1) predicts charge transfer excitation of a $\mathrm{Cu}$ hole into a ZRS near $\omega_{\mathrm{ZRS}}=2.25 \mathrm{eV}$. Its missing observation (at $T=300 \mathrm{~K}$ ) was ascribed to poor resolution ${ }^{1}$, or to uncoupled $\mathrm{CuO}_{4}$ units $\stackrel{2}{=}$ We show, that a ZRS visible at low $T$ would be strongly suppressed at $300 \mathrm{~K}$. However, if the Hamiltonian parameters are chosen to improve the description of $\mathrm{O} 1 s$ x-ray absorption $(\mathrm{XAS})^{7}$, optical, EELS1,2, and RIXS data ${ }^{5}$, then the ZRS absent at $T=0$, appears with rising $T$ but is accompanied by a Zhang-Rice triplet (ZRT) contribution at $\omega_{\mathrm{ZRT}} \approx 4 \mathrm{eV}$ in $\sigma(\omega)$ (superimposed with further transitions). The $\sigma(\omega)$ data are well described this way.

The used Hubbard Hamiltonian reads (cf. also Ref. 1)

$$
\mathcal{H}_{\mathrm{H}}=\mathcal{H}_{\mathrm{k}}+\mathcal{H}_{\mathrm{C}}+\mathcal{H}_{\text {ex }}
$$

Its kinetic, Coulomb, and exchange contributions are

$$
\begin{aligned}
\mathcal{H}_{\mathrm{k}} & =\sum_{i} \varepsilon_{i} n_{i}+\sum_{i, j, s} t_{i j} c_{i, s}^{\dagger} c_{j, s}, n_{i s}=c_{i s}^{\dagger} c_{i s} \\
\mathcal{H}_{\mathrm{C}} & =\sum_{i} U_{i} n_{i \uparrow} n_{i \downarrow}+\frac{1}{2} \sum_{i \neq j} V_{i j} n_{i} n_{j}, n_{i}=\sum_{s} n_{i s}, \\
\mathcal{H}_{\mathrm{ex}} & =\frac{1}{2} \sum_{i \neq j, s s^{\prime}} K_{i j}\left(c_{i s}^{\dagger} c_{j s^{\prime}}^{\dagger} c_{i s^{\prime}} c_{j s}+c_{i s}^{\dagger} c_{i s^{\prime}}^{\dagger} c_{j s^{\prime}} c_{j s}\right),
\end{aligned}
$$

where $i$ and $j$ run over all Cu- $3 d_{x y}$ and $\mathrm{O}-2 p_{x, y}$ orbitals and $s$ is the spin index. Except for the $\varepsilon_{i}$ the Hamiltonian parameters are the same as in Ref. 1: $U_{d}=8.5 \mathrm{eV}$, $U_{p}=4.1 \mathrm{eV}$ for the intra-orbital and $V_{p_{x}, p_{y}}=U_{p}-$ $2 K_{p_{x}, p_{y}}=2.9 \mathrm{eV}$ for the $\mathrm{O}$ onsite inter-orbital repulsion, where $K_{p_{x}, p_{y}}=0.6 \mathrm{eV}$ is the FM Hund's rule coupling. 
As in Ref. 1 isotropy of the $\mathrm{FM} \mathrm{Cu}-\mathrm{O}$ exchange integrals $K_{p d}=K_{p_{x} d}=K_{p_{y} d}=0.05 \mathrm{eV}$ was used for the sake of simplicity. Polarized O $1 s$ XAS measurements? with the electric field vector in $x$ and $y$ direction, respectively, indicated a nearly isotropic $\mathrm{O} 2 p$-hole distribution in the $x y$-plane. This indicates a condition $n_{p_{x}} \approx n_{p_{y}}$ within XAS error bars for the $\mathrm{O} 2 p$ hole occupation numbers in the GS. In order to achieve this despite the anisotropic $\mathrm{CuO}_{4}$ plaquette geometry, $\varepsilon_{p_{x}}-\varepsilon_{p_{y}}=0.2 \mathrm{eV}$ was taken as distinct from Ref. 1 where $\varepsilon_{p_{x}}=\varepsilon_{p_{y}}$ was assumed. In order to reproduce the first strong peak in $\sigma(\omega)$ near $4.4 \pm 0.2 \mathrm{eV}$ (Figs. 1,2), the mean $\mathrm{O}$ onsite energy $\Delta_{p d}=\left(\varepsilon_{p_{x}}+\varepsilon_{p_{y}}\right) / 2-\varepsilon_{d}$ has additionally been up-shifted by 0.5 to $3.7 \mathrm{eV}$. To demonstrate the strikingly distinct $\sigma(\omega)$ caused by these moderate changes, calculations were performed with the $\varepsilon_{i}$-values of Ref. 1, too. Hereafter these two models (2) are called M1 1 and M2 (above choice). We will show that they lead to different magnetic GS yielding different T-behavior of $\sigma(\omega)$.

Naturally, the dominating exchange integrals $J_{1}$ and $J_{2}$ determine the spectrum of low-energy excited states $|\nu\rangle$ (spin excitations) of the spin model (1). We found the $J$ values from projecting the Hamiltonian (2) onto (1). At a given $T$ and possibly in the presence of a magnetic field $H$, the optical conductivity $\sigma(\omega, H, T)$ of (2) is obtained from the $\sigma_{\nu}(\omega)$ with the initial spin states $|\nu\rangle$ of $(2)^{15}$ :

$$
\begin{aligned}
\sigma(\omega, T) & =\sum_{\nu} w_{\nu}(T)\left[1-\exp \left(-\hbar \omega / k_{\mathrm{B}} T\right)\right] \sigma_{\nu}(\omega), \\
w_{\nu} & =\frac{g_{\nu} \exp \left(-E_{\nu} / k_{\mathrm{B}} T\right)}{\sum_{\nu^{\prime}} g_{\nu^{\prime}} \exp \left(-E_{\nu^{\prime}} / k_{\mathrm{B}} T\right)}
\end{aligned}
$$

where for $H=0$ the spin degeneracy is $g_{\nu}=2 S_{\nu}+1$. For instance, for the largest cluster, $\mathrm{Cu}_{6} \mathrm{O}_{14}$, which can still be handled by the exact diagonalization of the Hamiltonian (2) there are $2^{6}=64$ low-energy spin states: $5 \times(S=$ $0), 9 \times(S=1), 5 \times(S=2)$, and $1 \times(S=3)$ multiplets. Due to the large optical transition energies $\hbar \omega \gg k_{\mathrm{B}} T$ the thermal occupation of the final states in (6) can be ignored. In case of an applied external magnetic field $H$ the $S \neq 0$ states with finite $S_{z}$ are Zeeman split which affects the Boltzmann probability $w_{\nu}=w_{\nu}(T, H)$ to find a cluster in a given spin state $\left(S_{\nu}, S_{z, \nu}\right)$. In this case the $g_{\nu}$ are replaced by

$$
\begin{aligned}
& g_{\nu}(H, T)=1+2 \sum_{\nu^{\prime}=1}^{\nu_{\max }} \cosh \left[\frac{\nu^{\prime} g_{\mathrm{L}} \mu_{\mathrm{B}} H}{k_{\mathrm{B}} T}\right] \\
& g_{\nu}(H, T)=2 \sum_{\nu^{\prime}=1}^{\nu_{\max }} \cosh \left[\frac{\left(2 \nu^{\prime}-1\right) g_{\mathrm{L}} \mu_{\mathrm{B}} H}{2 k_{\mathrm{B}} T}\right]
\end{aligned}
$$

for even and odd chains, respectively, where $g_{\mathrm{L}}$ denotes the Landé-factor. All response functions reported below were calculated using exact diagonalizations and the common continued fraction method for $\mathrm{Cu}_{n} \mathrm{O}_{2 n+2}$ clusters. The $\delta$-functions of the calculated $\sigma(\omega)$-spectra are convoluted with a Lorentzian broadening of $\gamma_{L}=0.35 \mathrm{eV}$ at half width to compare them with optical data.
Generally, the calculated $\sigma(\omega)$ for $\mathrm{Cu}_{n} \mathrm{O}_{2 n+2}$ chain clusters at $H=T=0$ exhibit a multiple peak structure: two well-pronounced peaks near 4 and 5 to $5.5 \mathrm{eV}$ as shown in Fig. 1. Remarkably, there are marked differences in $\sigma(\omega)$ between 2 and $4 \mathrm{eV}$ depending whether the GS is low-spin (i.e. a spin spiral) or high spin (i.e. FM). For $\mathrm{Li}_{2} \mathrm{CuO}_{2}$, due to its closeness to the critical point $\alpha_{c}$ (i.e. the FM-spiral transition), the issue depends sensitively on the $J$-values governed by the details of the parameters $\varepsilon_{i}$. A main issue of our analysis is that while the $\varepsilon_{i}$ values of M1 lead to a low-spin GS (spiral, $\alpha>\alpha_{c}$ ) our choice M2 results in a high-spin GS (FM, $\left.\alpha<\alpha_{c}\right) \cdot \sigma(\omega)$ for a $\mathrm{Cu}_{6} \mathrm{O}_{14}$ chain with assumption of a high-spin GS $(\mathrm{S}=3)$ or a low-spin GS $(\mathrm{S}=0,1)$ are shown on Fig. 2. The height of the feature between 2 and $3 \mathrm{eV}$ depends on the cluster size and on the total spin of the GS. Already a single $\mathrm{CuO}_{4}$ unit shows a double peak structure derived from transitions between an even parity singly occupied hybridized $\mathrm{Cu} 3 d \mathrm{O} 2 p$ state and odd parity non-bonding $\mathrm{O}$ states. Like the GS, all states are spin doublets. Since these transition energies do almost coincide with the mentioned above "high-energy" peaks for larger clusters, we regard them as intra-plaquette excitations. Inter-plaquette transitions may occur starting from dimers $n=2$. If their GS is an $S=0$ state or a low-spin state for larger clusters (e.g. a doublet for odd chains), a peak appears between 2 and $3 \mathrm{eV}$. This transition is forbidden, if the GS is the maximal spin state: i.e. an $S=n / 2$ FM state. Since in the optical transition $S$ and $S_{z}$ are conserved, the final state for an excited dimer in the former case is again a singlet (low-spin state) with two holes at one of the two $\mathrm{CuO}_{4}$ units, one sitting mainly on $\mathrm{Cu}$ and one sitting mainly on $\mathrm{O}$ (similar to a ZRS state on a $\mathrm{CuO}_{4}^{-5}$ unit). This transition is usually denoted as a ZRS excitation. In the FM case the excited state may contain a triplet (ZRT) of two holes on one plaquette, which in the optical excitation process occurs slightly below the main peak near $4 \mathrm{eV}^{16}$. The intensities of the ZRS and ZRT transitions exhibit some positive finite size effect $\propto 1 / n$ reflecting mainly the number of available plaquettes for an inter-plaquette transition. For a dimer with $J_{1}<0$ the GS is always a triplet since $J_{2}$ is involved for $n \geq 3$, only. A detailed finitesize analysis will be given elsewhere. The ZRT-energy $\approx$ $4 \mathrm{eV}$ found above is in excellent agreement with $4.1 \mathrm{eV}$ reported in a recent RIXS study $\underline{\underline{5}}$ but in sharp contrast with the assignment given in Ref. 4 where the RIXS peak near $2.1 \mathrm{eV}$ has been ascribed to the ZRS using a lowspin GS. Probably, the $2.1 \mathrm{eV}$-peak should be ascribed to $d$ - $d$ excitations 10,11 . A similar feature has been found in $\mathrm{CuGeO}_{3}$ (in $\sigma(\omega)$ at lower $\omega \approx 1.7 \pm 0.1 \mathrm{eV}$ ) while the ZRS is clearly observed at $3.4 \pm 0.2 \mathrm{eV} \underline{17,18}$.

Exact diagonalization of (2) yields excitation energies $\omega_{\mathrm{ZRS}}=2.7 \mathrm{eV}, \omega_{\mathrm{ZRT}}=4 \mathrm{eV}$ for $\mathrm{M} 2$ and $\omega_{\mathrm{ZRS}}=2.25 \mathrm{eV}$, $\omega_{\mathrm{ZRT}}=3.7 \mathrm{eV}$ for M1. For both models this is consistent with $\Delta_{\mathrm{ZR}} \approx 1.3 \mathrm{eV}$ obtained as the distance between the lowest $S=0$ and the $S=1$ levels in a single $\mathrm{CuO}_{4}^{-5}$ plaquette with two holes. The $\sigma(\omega, T)$ obtained from (7) 


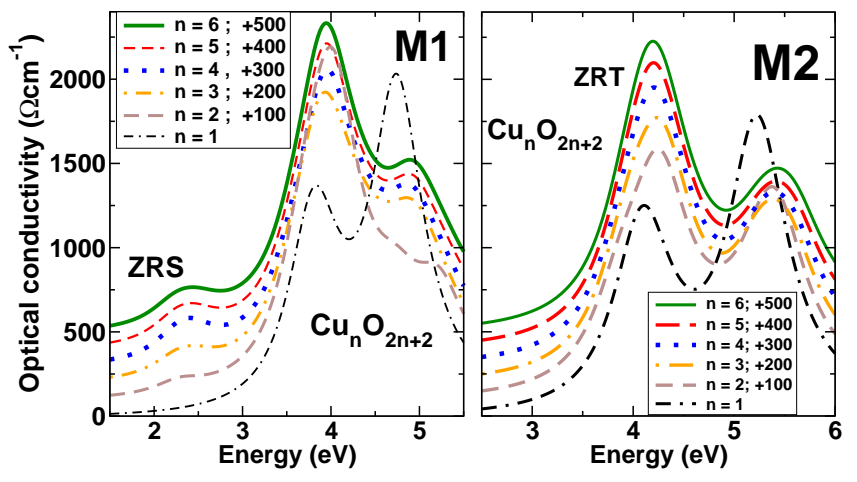

FIG. 1: (Color). Finite size effect for $\sigma(\omega)$. Left (right): GS given by the lowest (highest) spin state. The curves for various clusters are shifted by $(\mathrm{n}-1) \times 100(\Omega \mathrm{cm})^{-1}$.

for both models are shown on Fig. 3, upper two panels. At $300 \mathrm{~K} \mathrm{M} 1$ and M2 yield the same qualitative behavior: the ZRS is largely suppressed. The main peak position of the experiment at $\gtrsim 4 \mathrm{eV}$ is, however, much better reproduced by M2. We studied also the field dependence of $\sigma(\omega, T, H)$ (Fig. 3, lower panel) and found it similar in both models. To repeat it, M1 results in $-J_{2} / J_{1}=\alpha>\alpha_{c}$ when projected onto the spin Hamiltonian (1) and hence in a low-spin GS while M2 resulting in $\alpha<\alpha_{c}$ and hence in a FM GS. The reason for the reduction of $\alpha$ is mainly a reduction in superexchange due to the enhanced $\Delta_{p d}$-value. As is clearly seen from Fig. 3, whether $\alpha<\alpha_{c}$ or $\alpha>\alpha_{c}$ can directly experimentally be decided by low- $T$ studies of $\sigma(\omega)$ and such measurements are strongly encouraged. In this context we stress once more that our main result is not simply the determination of a new, improved, parameter set M2 in a literal sense, but the demonstration of an unique correlation between the magnetic nature of the GS and the $T$-dependence of $\sigma(\omega)$. A refinement of M2 when low- $T$ and $H \neq 0$ data will be available, a generalization of the Hamiltonian (2) to include further orbitals or interactions, its application

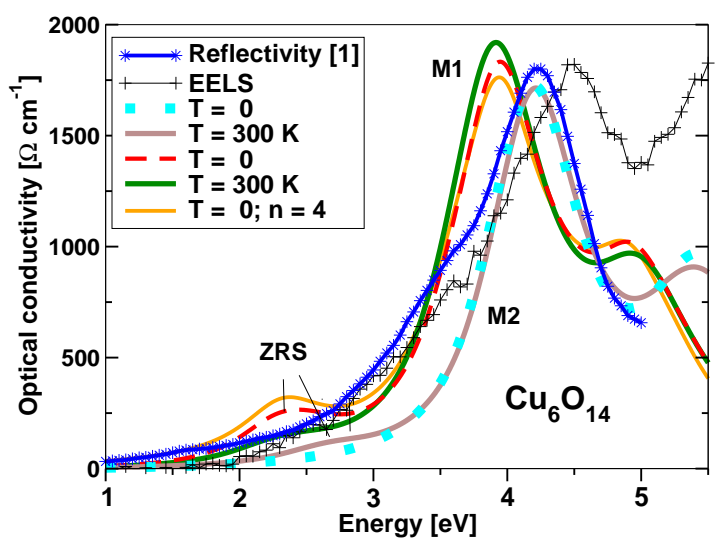

FIG. 2: (Color). Experimental and theoretical optical inchain conductivities of (2) for $T=0$ and $T=300 \mathrm{~K}$ using a low-spin $(S=0,1)$ GS (M1) and a high-spin $(S=3)$ GS (M2).

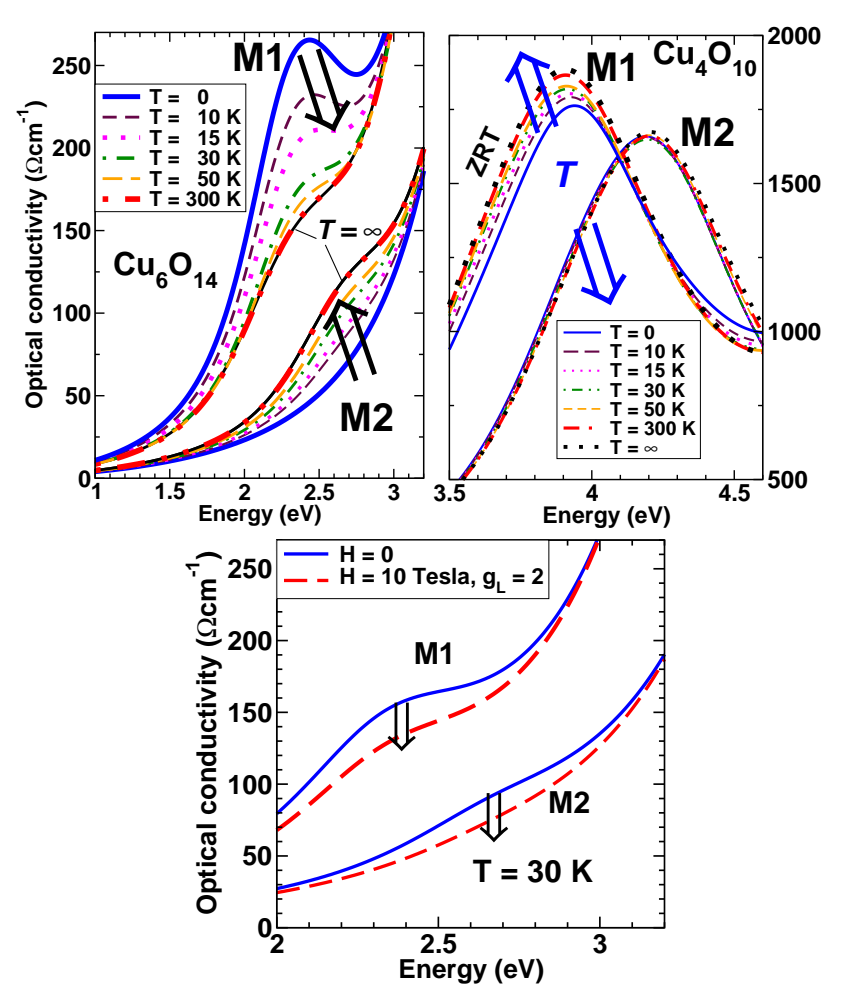

FIG. 3: (Color). T-dependent optical conductivity at $H=0$ (upper) and at $H \neq 0$ (lower) within the regions of ZRS and ZRT transitions for clusters within a low-spin (M1) and a high-spin GS (M2). ' $T=\infty$ ' means $k_{\mathrm{B}} T \gg\left|J_{i}\right|$, but still $\hbar \omega \geq 10^{4} \mathrm{~K} \gg k_{\mathrm{B}} T$.

to other cuprates like $\mathrm{Li}_{2} \mathrm{ZrCuO}_{4}$ with a different set will not change this fundamental interrelation.

To compare the $J_{i}$ with those from another method, density functional theory (DFT) calculations for $\mathrm{Li}_{2} \mathrm{CuO}_{2}$ using the structural data of Ref. 5 were performed in the local spin density plus Hubbard $U$ $(\mathrm{LSDA}+U)$ approach. The full-potential local-orbital (FPLO) code ${ }^{19}$ was employed. The onsite Coulomb integral for a $\mathrm{Cu} 3 d_{x y}$ hole was taken to be $U=8 \mathrm{eV}$ and an onsite exchange integral $J=1 \mathrm{eV}$ was used. The $\mathrm{LSDA}+U$ yields quite reliable results for total energy differences of different spin structures from which the $J_{i^{-}}$ values of Eq. (1) can be extracted. TAB. I shows the $J_{1}$ and $J_{2}$-values we found in comparison with similar results given in Refs. 9, 14 incl. a quantum chemistry study of di- and trimers, with those from projecting the models M1 and M2 onto Eq. (1). The small $J_{3}$-values justify a description of $\mathrm{Li}_{2} \mathrm{CuO}_{2}$ in terms of a $J_{1^{-}} J_{2}$ model. There is an almost perfect agreement of three independent approaches to be close to $\alpha_{c}=0.25\left(J_{3}=0\right)$ of a quasi-1D situation. Moreover, model M2, the LSDA $+U$ as well as the QC calculations point to $\alpha<\alpha_{c}$, i.e. to a FM chain. This holds in our LSDA $+U$, if $7 \mathrm{eV} \leq U \leq 9 \mathrm{eV}$.

To conclude, we have shown that the model manybody Hamiltonian (2) with parameters M2 is capable of consistently describing XAS, EELS, RIXS and $\sigma(\omega)$ of 
TABLE I: In-chain exchange integrals $J_{i}$ and frustration ratio $\alpha=-J_{2} / J_{1}$ obtained from exact diagonalizations (ED) of the Hubbard model (2) with sets M2 and M1 as well as from independent $\mathrm{DFT}+U$ and a quantum chemistry (QC) study.

\begin{tabular}{|l|c|c|c|c|c|}
\hline & \multicolumn{2}{|c|}{ present work } & \multicolumn{3}{|c|}{ other work } \\
& $\mathrm{ED}^{a}$ & $\mathrm{DFT}^{b}$ & $\mathrm{ED}^{c}$ & $\mathrm{DFT}^{d}$ & $\mathrm{QC}^{e}$ \\
\hline$J_{1}, \mathrm{~K}$ & -146 & -154 & -103 & -126 & -142 \\
$J_{2}, \mathrm{~K}$ & 33 & 30 & 49 & 52 & 22 \\
$J_{3}, \mathrm{~K}$ & -0.5 & - & -2 & - & - \\
$\alpha$ & 0.23 & 0.19 & 0.47 & 0.41 & 0.15 \\
\hline \hline
\end{tabular}

${ }^{a} \mathrm{M} 2, \mathrm{Cu}_{4} \mathrm{O}_{10}$ chain cluster

${ }^{b} \mathrm{LSDA}+U$ (FPLO), $U=8 \mathrm{eV}, J=1 \mathrm{eV}$

${ }^{c} \mathrm{M} 1$ [1]; $\mathrm{Cu}_{4} \mathrm{O}_{10}$ chain cluster

${ }^{d}$ GGA $+U($ WIEN $2 k) ; U_{\text {eff }}=U-J=8 \mathrm{eV}$, see Ref. 14

$e$ TAB. II of Ref. 9

$\mathrm{Li}_{2} \mathrm{CuO}_{2}$ at $300 \mathrm{~K}$. The main issue, however, is that the magnetic GS and the spin excitation spectrum strongly affect the $T$-dependence of $\sigma(\omega)$ in the visible range. The reason for is the thermal population of excited spin states which differ magnetically much from the GS. This qualitatively new effect, irrespectively on details of the relevant microscopic parameters for a particular compound, has been overlooked so far in the cuprate optics literature. Our findings allow a quick qualitative magnetic classification of cuprates by optical measurements: if the AFM (FM) exchange is dominant, $\sigma(\omega)$ in the ZRS energy region increases (decreases) lowering $T$. In the 1D FM- $J_{1}-\mathrm{AFM}-J_{2}$ case it even allows a sharp determination of the position of a system relative to the critical point. Thus, a $T$-dependent $\sigma(\omega)$ owing to thermally activated spin excitations is a general phenomenon. Experimental studies of this $T$-dependence together with a quantitative theoretical analysis of many-body models can provide deep insight in the GS and the spin excitation spectrum of undoped insulators. This is relevant also for weakly coupled systems with un-shared $\mathrm{CuO}_{4}$-plaquettes such as $\mathrm{Bi}_{2} \mathrm{CuO}_{4}{ }^{20}$ or $\mathrm{Sr}_{2} \mathrm{Cu}(\mathrm{PO})_{4}^{21}$. For $\mathrm{Li}_{2} \mathrm{CuO}_{2}$ such studies can be decisive to settle the intra-chain exchange and the reason for the FM in-chain order. If $\alpha_{c}>\alpha$ would be true and the FM in-chain order below $T_{N}$ would be due to weak inter-chain exchange, a non-monotonic behavior of $\sigma(\omega, T)$ above $T_{N}$ should be observed. For $\mathrm{La}_{6} \mathrm{Ca}_{8} \mathrm{Cu}_{24} \mathrm{O}_{41}$ and $\mathrm{Y}_{2} \mathrm{Ca}_{2} \mathrm{Cu}_{5} \mathrm{O}_{10}$ also showing FM inchain order a similar situation can be anticipated. Near a critical point as in $\mathrm{Li}_{2} \mathrm{ZrCuO}_{4}{ }^{13}$ also the magnetic field dependence becomes an issue.

We thank the DFG (Dre-269/3-1;D,M), its EmmyNoether program (R), the ASCR project AVOZ10100520 (M), the GIF (M,R,E) for support, and M. Knupfer, R. Kuzian, J. Richter, and S. Nishimoto for discussions.

Note added. After submission of the present paper $\sigma(\omega, T)$ for $\mathrm{Sr}_{2} \mathrm{CuO}_{3}$ has been studied by Kim et al. (arXiv:0806.2083). There (see Fig. 1) a slight reduction of the intensity of the charge transfer transition (CT) near $1.8 \mathrm{eV}$ by raising $T$ has been found, similarly to what we have shown in Fig. 3. In our approach this behavior is attributed to optical excitations from thermally populated low-lying states which exhibit a reduced $\sigma(\omega)$ in the mentioned above $\mathrm{CT}$ region. For long chains the existence of such states (e.g. triplets) independently on the value of $J_{1}>0$ follows from the Bethe-ansatz solution of the related 1D-AFM spin-1/2 Heisenberg model.
* Corr. author; electr. address: drechsler@ifw-dresden.de

1 Y. Mizuno, T. Tohyama, S. Maekawa et al., Phys. Rev. B 57, 5326 (1998).

2 S. Atzkern, M. Knupfer, M. Golden et al., ibid. 62, 7845 (2000).

3 S.-L. Drechsler, J. Richter, J. Málek, et al., J. Mag. \& Mag. Mat. 290, 345 (2005); J. of Phys. Cond. Mat. 19, 145230 (2007).

4 F. Vernay, B. Moritz, I. Efimov et al., Phys. Rev. B 77, 104519 (2008).

${ }^{5}$ T. Learmonth, C. McGuinness, P.A. Glans et al., Europhys. Lett. 79, 47012 (2007).

${ }^{6}$ F. Sapina, J. Rodriguezcarvajal, M.J. Sanchis et al., Sol. Stat. Comm. 74, 779 (1990).

7 R. Neudert, H. Rosner, S.-L.Drechsler et al., Phys. Rev. B 60, 13413 (1999).

8 Z. Hu, S.-L. Drechsler, J. Málek et al., Europhys. Lett. 59, 135 (2002).

${ }^{9}$ C. de Graaf, I. de P. R. Moreira, F. Illas, O. Iglesias, and A. Labarta, Phys. Rev. B 66, 014448 (2002).

10 M. Hasan, Y. Li, Y. Chuang et al., Int. J. of Mod. Phys. 17, 3519 (2003).

11 Y. Kim, J. Hill, F. Chou et al., Phys. Rev. B 69, 155105
(2004).

12 E.M.L. Chung, G.J. McIntyre, D.M. Paul, G. Balakrishnan, and M.R. Lees, ibid. 68, 144410 (2003).

13 S.-L. Drechsler et al., Phys. Rev. Lett. 98, 077202 (2007).

14 H.J. Xiang, C. Lee and M.-H. Whangbo, Phys. Rev. B 76, 220411(R) (2007).

15 Similar finite $T$-approaches have been used for the $t-J$ model e.g. by J. Jaklič and P. Prelovšek, Adv. Phys. 19, 1 (2000), for spin-less fermions by X. Zotos and P. Prelovšek, Phys. Rev. B 53, 983 (1996), and for single-band Hubbard models at $U \gg t$ by $\mathrm{H}$. Onodera, T. Tohyama, and S. Maekawa, ibid. 69, 245117 (2004).

16 This notation reflects properly only the excitation energies. There is no change of $S_{z}$ or $S$ of a chain (cluster) as the term "triplet" excitation might suggest.

17 F. Bondino, M. Zangrando, M. Zacchigna et al., Phys. Rev. B 75, 195106 (2007).

18 S. Pagliara, F. Parmigiani, P. Galinetto, A. Revcolevschi, and G. Samoggia, ibid. 66, 024518 (2002).

19 K. Koepernik and H. Eschrig, ibid. 59, 1743 (1999). Here we used the code FPLO 5.00-19 and the basis set of Ref. 13 .

20 O. Janson, R. Kuzian, S.-L. Drechsler, and H. Rosner, ibid. 76, 115119 (2007). 
21 M. Johannes, J. Richter, S.-L. Drechsler, and H. Rosner, ibid. 74, 174435 (2006). 